\title{
Zero-Hopf Bifurcation in the Generalized Stretch-Twist-Fold Flow
}

\author{
Niazy H. Hussein ${ }^{1 *}$ and Azad I. Amen ${ }^{2}$
}

${ }^{1}$ Mathematics Department, Faculty of Science, Soran University, Soran-Erbil Kurdistan Region, Iraq. Mathematics Department, College of Basic Education, Raparin University, Ranya, Kurdistan Region, Iraq; ${ }^{2}$ Salahaddin University, Basic Education College, Mathematics Department, Kirkuk Road, Erbil, Iraq. College of Science, Knowledge University, Erbil Kurdistan Region, Iraq. ${ }^{\star}$ Email: niazy.hussein@maths.soran.edu.krd

\begin{abstract}
A zero-Hopf equilibrium in a three-dimensional system is an isolated equilibrium point which has a zero eigenvalue and a simple pair of purely imaginary eigenvalues. In general, for such an equilibrium, there is no theory for finding when some periodic solutions are bifurcated by perturbing the parameters of the system. In this work, we describe the values of the parameters for which a zero-Hopf equilibrium occurs at the equilibrium points in the generalized stretch-twist-fold flow. Thus, only one condition for parameters of the generalized stretch-twist-fold flow introduced in a system (Eq. 1) is found for which the equilibrium point is a zero-Hopf equilibrium. For this condition, we use the averaging method to provide the existence of a periodic solution, which bifurcates from the zero-Hopf equilibrium point. The main result in this paper is Theorem 1, which gives a periodic solution of the generalized stretch-twist-fold flow.
\end{abstract}

Keywords: Stretch-twist-fold flow; Zero-Hopf equilibrium point; Zero-Hopf bifurcation; Periodic solution; The averaging method.

$$
\text { تشعب هوبف الصفري في إمتدادـ إلتواءـ التدفق المطوي المعمم }
$$

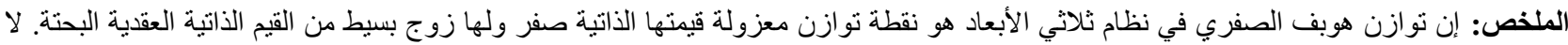

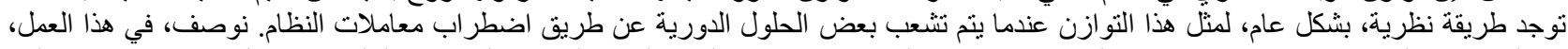

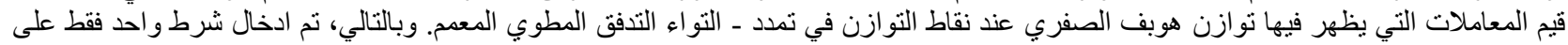

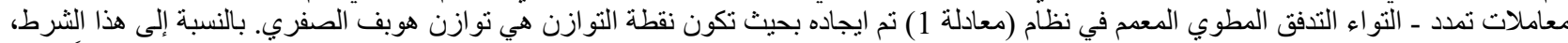

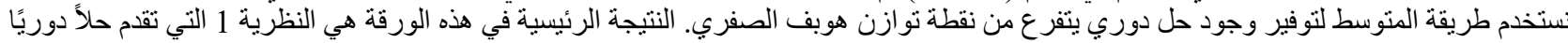
لتمدد ـ التو اء التدفق المطوي المعدم. الكلمات المفتاحية: امتداد ـ التواء ـ التدفق المطوي، نقطة توازن هوبف الصفري، تشعب هوبف الصفري، الحل الدوري ، طريقة المتوسط.

\section{Introduction}

s a special case of Stokes flows, the stretch-twist-fold (STF) flow normally appears in dynamo theory. This flow was first formulated in the work of Vainshtein and Zel'dovich [1]. On the principle of this research, Moffatt in [2] invented a particular quadratic flow to represent the STF action. There are also very extensive studies of the STF flow; one can see [3-8]. Dependent on the STF flow, a generalized stretch-twist-fold (GSTF) flow was suggested in [9] by Jianghong and Qigui. They added two extra parameters in the STF flow in order to further investigate the STF system. The parameters for which the Hopf equilibrium point occurs in the GSTF flow are described in [9]. The new parameter not only preserves the original dynamic properties but also has some novel characteristics. 
Now, in the following, the GSTF system is considered

$$
\left.\begin{array}{l}
\dot{x}=\mathrm{a}_{1} \mathrm{z}-8(\mathrm{~b}+1) \mathrm{xy}, \\
\dot{y}=11 \mathrm{x}^{2}+3 \mathrm{y}^{2}+\mathrm{z}^{2}+\mathrm{a}_{2} \mathrm{xz}-3 \mathrm{c}^{2}, \\
\dot{z}=-\mathrm{a}_{1} x+2 y z-\mathrm{a}_{2} x y,
\end{array}\right\}
$$

where $\mathrm{a}_{1}$ and $\mathrm{a}_{2}$ are real positive parameters and are associated with the ratios of intensities of the SFT ingredients of the flow, and $b, c$ are real parameters added in the original STF flow which determines the bifurcations of the system. Moreover, an over-dot indicates the derivative with respect to independent variable $t$. System (1) remains nonconservative anymore $(\nabla V=-8 b y)$, and the original STF flow does not exhibit the Hopf bifurcation, but the authors in [1] showed that system (1) has the Hopf bifurcation, which is very interesting. They used the normal form theory for studying Hopf bifurcations in detail. The authors in [9] proved that system (1) has no Darboux first integral when $\mathrm{a}_{1} \neq 0, b=0$ and $c= \pm 1$.

In this work, our main goal is to study the existence of the zero-Hopf equilibria; we put a suitable condition on the singular point to obtain a zero-Hopf equilibrium point, and which gives the zero-Hopf bifurcations in the GSTF flow (1). This is described in Proposition 1. Generally, for this kind of equilibrium there is no theory to explain when it bifurcates some periodic solutions which move the parameters of the system. We then introduce a suitable rescaling of variables which transform the GSTF system (1) into the normal form system; the condition for the existence of a periodic solution which bifurcates from the zero-Hopf equilibrium is given by using the averaging method. This method will be given in Section 4 also contains some basic results to obtain a periodic solution of three dimensional polynomial differential systems. This investigation provides a new results for a periodic solution of the GSTF flow, which we have not found in the literature. Whether or not a system has periodic solutions is very interesting and important in non-linear differential systems. The existence of periodic solutions for this kind of bifurcation in a 3D differential system has been widely addressed in [10-17].

Actually, in [1] the authors investigate the Hopf bifurcation of system (1). Their results are more general than the present ones, but our results are more accurate for finding periodic solution produced from a zero-Hopf equilibrium.

The reason is that we follow the conditions of the averaging method which is described in Theorem 2. These conditions clearly guarantee that there is a periodic solution of the given system.

When the GSTF system (1) possessing a zero-Hopf equilibrium point at the origin of coordinates possess a zeroHopf bifurcation, it produces some periodic solutions. For performing this, we consider $\epsilon$-perturbations of the values of the parameters for which new ones have a zero-Hopf equilibrium. The small parameter $\epsilon$ is necessary to be used in the averaging method, and the analysis of a zero-Hopf bifurcation will be executed with respect to it.

This work is arranged as follows. In Section 2, the equilibrium of system (1) is investigated. In Section 3 , the explicit parametric condition of existence of a zero-Hopf equilibrium and a periodic solution of system (1) is given. Section 4 explains the averaging method of the first order. In Section 5, we give the proofs of the results presented in Section 3, and we also describe the stability or instability of the periodic solution which is produced in such a zeroHopf bifurcation of the GSTF system (1). The last section concludes the work.

\section{Equilibrium}

The equilibria of system (1) for $a_{1} \neq 0$ are all on the following surface of the ellipsoid

$$
\left(1-\frac{8}{3} b\right) x^{2}+y^{2}+z^{2}=c^{2}
$$

We obtain (2) by solving the following three equations

$$
a_{1} z-8(b+1) x y=0, \quad 11 x^{2}+3 y^{2}+z^{2}+a_{2} x z-3 c^{2}=0, \quad-a_{1} x+2 y z-a_{2} x y=0 .
$$

For $a_{1}=0$, the equilibria are the solutions of the following equations

$$
8(b+1) x y=0, \quad 11 x^{2}+3 y^{2}+z^{2}+a_{2} x z-3 c^{2}=0, \quad 2 y z-a_{2} x y=0 .
$$

If $y=0$, the equilibria are a quadratic curve

$$
11 x^{2}+z^{2}+a_{2} x z-3 c^{2}=0, \quad y=0,
$$

If $y \neq 0$, then the two isolated points $E_{+}(0, c, 0)$ and $E_{-}(0,-c, 0)$ are the equilibria. We are only interested in the case where $a_{1} \neq 0$ and $c=0$. According to equation (2), these give us the origin of coordinates as the only equilibrium point of system (1). 


\section{HUSSEIN, N.H. and AMEN, A.I.}

\section{Statement of the main results}

If an isolated equilibrium point of a 3D autonomous differential system has a zero eigenvalue and a pair of purely imaginary eigenvalues then this kind of equilibrium point is called the zero-Hopf equilibrium (also called a saddlenode Hopf bifurcation). It comes out that its unfolding owns the rich dynamics in a small neighborhood of the origin. This means that, unfolding is used to describe the dynamics in small neighborhoods of the equilibria and their perturbations (see for instance, Guckenheimer [18], Guckenheimer and Holmes [19], Scheurle and Marsden [20], Kuznetsov [21], and the references therein). In some cases a zero-Hopf bifurcation may lead to a local birth of "chaos" as can be found in the work of Baldom'a and Seara [22], and Broer and Vegter [23].

Our goal in the next proposition is to find a condition in which a zero- Hopf equilibrium point takes the place of system (1).

Proposition 1. There is only a one-parameter family of system (1) in which the equilibrium point localized at the origin of coordinates is a zero-Hopf equilibrium point. That is

$$
c=0 .
$$

We will prove this proposition in Section 4. This condition is the only one-parameter family of system (1) which exhibits a zero-Hopf equilibrium localized at the equilibrium point.

The next theorem tells us there is a small amplitude periodic solution which bifurcates from a zero-Hopf equilibrium point of system (1).

Theorem 1. Let the vector given by $\left(a_{1}, a_{2}, b, c\right)=\left(\tilde{a}_{1}+\epsilon \alpha_{1}, \tilde{a}_{2}+\epsilon \alpha_{2}, \tilde{b}+\epsilon \alpha_{3}, \epsilon \alpha_{4}\right)$ with $\epsilon>0$ a sufficiently small parameter. If

$$
\alpha_{4} \neq 0 \text {, and } \tilde{b} \neq \frac{3}{4} \text {. }
$$

then system (1) has a zero-Hopf bifurcation at the equilibrium point which localizes at the origin of coordinates, and when $\epsilon=0$, a limit cycle (periodic solution) is produced at this equilibrium. Furthermore, a stability or instability of this limit cycle is the same as the equilibrium point of a planar differential system with the following eigenvalues

$$
\pm \frac{1}{\omega} \sqrt{-6 \alpha_{4}^{2}(3+4 \tilde{b})}
$$

In Section 4, the above theorem will be proved via the averaging method of first order. This method is explained briefly in the following section.

\section{Periodic solutions via the averaging method}

In this work, we use the averaging method to study a periodic solution of the GSTF flow (1). The averaging method is a classical and developed tool to determine the behavior of the dynamics of non-linear differential systems, and in particular of their periodic orbits. This method has a long history which starts with the works of Lagrange and Laplace. An intuitive justification of the process was provided by them. The first formalization of this method is due to Fatou [24]. Bogoliubov and Krylov [25], and Bogoliubov [26], made important practical and theoretical contributions using this method.

In the following, the basic results concerning he averaging theory of the first order can be summarized as follows

Theorem 2. Consider the following two initial value problems:

and

$$
\dot{x}=\epsilon f_{1}(t, x)+\epsilon^{2} f_{2}(t, x, \epsilon), x(0)=x_{0},
$$

$$
\dot{y}=\epsilon g(y), \quad y(0)=x_{0},
$$

where $x, y, x_{0} \in \Omega$ is an open subset of $\mathbb{R}^{n}$, with $t \in[0, \infty)$. Furthermore, we suppose that $f_{1}(t, x)$ and $f_{2}(t, x, \epsilon)$ are periodic of T-period in the variable $t$, and $g(y)$ is the averaged function of $f_{1}(t, y) \in \Omega$. We mean that

Suppose

$$
g(y)=\frac{1}{T} \int_{0}^{\pi} f_{1}(t, y) d t
$$

1. $f_{1}, \frac{\partial f_{1}}{\partial x}, \frac{\partial^{2} f_{1}}{\partial x^{2}}, f_{2}$ and $\frac{\partial f_{2}}{\partial x}$ are all defined, continuous in $\mathbb{R}^{+} \times \Omega$, and these expressions are bounded by a constant independent of $\epsilon \in\left(0, \epsilon_{0}\right]$.

2. $\mathrm{T}$ is a constant independent $\epsilon$, and

3. $y(t)$ belongs to $\Omega$ on the interval of time $[0,1 / \epsilon]$. 


\section{ZERO-HOPF BIFURCATION IN THE GSTF FLOW}

Then the following statements hold

- On the time scale for $\frac{1}{\epsilon}$, we have that $x(t)-y(t)=O(\epsilon)$, as $t \rightarrow 0 \$$.

- If $\mathrm{p}$ is an equilibrium point of the averaged system (5) such that

$$
\operatorname{det}\left(\frac{\partial g}{\partial y}\right)_{y=p} \neq 0
$$

Then, there is a T-periodic solution $\phi(t, \epsilon)$ for system (4) which is close to p and such that $\phi(t, \epsilon)$ approaches to $p$, as $\epsilon$ approaches to zero. Furthermore, the equilibrium point of averaging differential system (5) has the same stability or instability as the periodic solution $\phi(t, \epsilon)$, and the equilibrium point with eigenvalues. Actually, the equilibrium point $p$ has the stability behavior of the Poincar'e map associated to the limit cycle $\phi(t, \epsilon)$. The proof of the averaging method of the first order to determine periodic solutions can be found in the book of Sanders and Verhulst [27], see Theorems 11.5 and 11.6.

\section{Proof of the main results}

Proof of Proposition 1: For $a_{1} \neq 0$ and $c \neq 0$, system (1) has two isolated equilibria $E_{ \pm}=(0, \pm c, 0)$, which collide at the origin coordinates where $c=0$. The proof is made by calculating directly the eigenvalues at the origin coordinates. Note that the linear part of system (1) has the following characteristic polynomial at the origin of coordinates

$$
p(\lambda)=\lambda^{3}+a_{1}^{2} \lambda=\lambda\left(\lambda^{2}+a_{1}^{2}\right) .
$$

Putting that $p(\lambda)=\lambda\left(\lambda^{2}+\delta^{2}\right)=0$, where $\delta=a_{1}>0$, thus the proposition follows.

Proof of Theorem 1: If we consider the $\epsilon$-perturbations $\left(a_{1}, a_{2}, b, c\right)=\left(\tilde{a}_{1}+\epsilon \alpha_{1}, \tilde{a}_{2}+\epsilon \alpha_{2}, \tilde{b}+\epsilon \alpha_{3}, \epsilon \alpha_{4}\right)$, with $\epsilon>0$ a sufficiently small parameter, then, system (1) becomes

$$
\left.\begin{array}{l}
\dot{x}=\left(\tilde{a}_{1}+\epsilon \alpha_{1}\right) z-8\left(\tilde{b}+\epsilon \alpha_{3}+1\right) x y, \\
\dot{y}=11 x^{2}+3 y^{2}+z^{2}+\left(\tilde{a}_{2}+\epsilon \alpha_{2}\right) x z-3 \epsilon \alpha_{4}^{2}, \\
\dot{z}=-\left(\tilde{a}_{1}+\epsilon \alpha_{1}\right) x+2 y z-\left(\tilde{a}_{2}+\epsilon \alpha_{2}\right) x y .
\end{array}\right\}
$$

By the rescaling of variables $(x, y, z)=(\epsilon X, \epsilon Y, \epsilon Z)$, system (8) in the new variables $(X, Y, Z)$ becomes

$$
\left.\begin{array}{l}
\dot{X}=\left(\tilde{a}_{1}+\epsilon \alpha_{1}\right) Z-8\left(\epsilon \tilde{b}+\epsilon^{2} \alpha_{3}+\epsilon\right) X Y \\
\dot{Y}=\epsilon Z^{2}+\epsilon\left(\tilde{a}_{2}+\epsilon \alpha_{2}\right) X Z+\epsilon\left(11 X^{2}+3 Y^{2}-3 \alpha_{4}^{2}\right), \\
\dot{Z}=-\left(\tilde{a}_{1}+\epsilon \alpha_{1}\right) X+2 \epsilon Y Z-\left(\epsilon \tilde{a}_{2}+\epsilon^{2} \alpha_{2}\right) X Y .
\end{array}\right\}
$$

Now, the linear part at the origin of system (9) can be written in its real Jordan normal form, namely when $\epsilon=0$, we shall transform the linear part at the origin of the differential equation (9) into its real Jordan normal form, which is as

$$
\left(\begin{array}{ccc}
0 & -\omega & 0 \\
\omega & 0 & 0 \\
0 & 0 & 0
\end{array}\right), \text { where } \omega=\tilde{a}_{1}>0
$$

For doing this we use the linear change of variables $(X, Y, Z) \rightarrow(U, V, W)$ given by

$$
(X, Y, Z)=P(U, V, W), \quad \text { where } P=\left(\begin{array}{ccc}
-1 & 0 & 0 \\
0 & 0 & 1 \\
0 & 1 & 0
\end{array}\right)
$$

Clearly, $P^{-1}$ exists. Then system (9) can be written in the new variables $(U, V, W)$ in the following

$$
\begin{gathered}
\dot{U}=-V \omega+\left((-8 \tilde{b}-8) U W-V \alpha_{1}\right) \epsilon-8 \epsilon^{2} U W \alpha_{3}, \\
\dot{V}=U \omega+\left(U \alpha_{1}+2 W V+U W \tilde{a}_{2}\right) \epsilon+\epsilon^{2} W \alpha_{2}, \\
\dot{W}=\left(11 U^{2}+3 W^{2}+V^{2}-U V \tilde{a}_{2}-3 \alpha_{4}^{2}\right) \epsilon-\epsilon^{2} U V \alpha_{2} .
\end{gathered}
$$

To study the periodic solutions of system (11) when $\epsilon$ is too small, we use the following a class of cylindrical coordinates

$$
(U, V, W)=(r \cos (\theta), r \sin (\theta), W), r>0 .
$$




\section{HUSSEIN, N.H. and AMEN, A.I.}

This is a topological change of variables in a deleted neighborhood of origin coordinates.

$$
\begin{gathered}
\dot{r}=\epsilon\left(2\left(\epsilon \alpha_{2}-\tilde{a}_{2}\right) \sin (\theta) \cos (\theta)+\left(8 \epsilon \alpha_{3}+8 \tilde{b}+10\right) \cos ^{2}(\theta)\right) r W, \\
\dot{\theta}=\omega+\epsilon \alpha_{1}+\epsilon\left(\left(10+8 \epsilon \alpha_{3}+8 \tilde{b}\right) \sin (\theta) \backslash \cos (\theta)+\left(\tilde{a}_{2}+\epsilon \alpha_{2}\right) \cos ^{2}(\theta)\right) W, \\
\dot{W}=-3 \epsilon \alpha_{4}^{2}+\epsilon\left(10 \cos ^{2}(\theta)-\left(\tilde{a}_{2}+\epsilon \alpha\right) \cos (\theta) \sin (\theta)\right) r^{2}+3 \epsilon W^{2},
\end{gathered}
$$

where $\omega=\tilde{a}_{1}>0$. This implies that there exists a small neighborhood of $(r, W)=(0,0)$ such that $\dot{\theta} \neq 0$ for all $\theta$ in this neighborhood. Furthermore, we consider an equivalent system of (12) in the neighborhood of $(r, W)=$ $(0,0)$, introducing also $\theta$ as the new independent variable. Thus, we obtain the following system

$$
\left.\begin{array}{l}
\frac{d r}{d \theta}=\epsilon, f_{1,1}+\frac{\epsilon^{2}}{\omega^{2}} f_{2,1}(r, \theta, W)+O\left(\epsilon^{3}\right), \\
\frac{d W}{d \theta}=\epsilon f_{1,2}+\epsilon^{2} f_{2,2}(r, \theta, W)+O\left(\epsilon^{3}\right),
\end{array}\right\}
$$

where

$$
\begin{aligned}
& f_{1,1}=-r \frac{W}{\omega}\left(8(\cos (\theta))^{2} \tilde{b}+10(\cos (\theta))^{2}-2-\sin (\theta) \cos (\theta) \tilde{a}_{2}\right), \\
& f_{1,2}=\frac{1}{\omega}\left(-r^{2} \cos (\theta) \sin (\theta) \tilde{a}_{2}+3 W^{2}-3 \alpha_{4}^{2}+r^{2}+10 r^{2}(\cos (\theta))^{2}\right), \\
& f_{2,1}=\left(8\left(\omega \alpha_{3}-\tilde{b} \alpha_{1}\right) \cos ^{2}(\theta)+\sin (\theta) \cos (\theta) \tilde{a}_{2} \alpha_{1}+2 \alpha_{1}-\cos (\theta) \sin (\theta) \alpha_{2} \omega-10 \cos ^{2}(\theta) \alpha_{1}\right) r W \\
& \quad+\left(-160 \cos ^{3}(\theta) \sin (\theta) \tilde{b}+20 \sin (\theta) \cos (\theta)+12 \cos ^{2}(\theta) \tilde{a}_{2}+\cos ^{3}(\theta) \sin (\theta) \tilde{a}_{2}^{2}\right. \\
& \quad+8 \cos ^{2}(\theta) \tilde{a}_{2} \tilde{b}-64 \cos ^{3}(\theta) \tilde{b}^{2} \sin (\theta)-16 \cos ^{4}(\theta) \tilde{a}_{2} \tilde{b}-20 \cos ^{4}(\theta) \tilde{a}_{2} \\
& \left.\quad-100 \cos ^{3}(\theta) \sin (\theta)+16 \sin (\theta) \cos (\theta) \tilde{b}\right) W^{2} r \\
& f_{2,2}=3 \alpha_{1} \alpha_{4}^{2}+ \\
& \quad\left(3 \alpha_{4}^{2} \cos ^{2}(\theta) \tilde{a}_{2}+30 \alpha_{4}^{2} \sin (\theta) \cos (\theta)+24 \alpha_{4}^{2} \sin (\theta) \cos (\theta) \tilde{a}\right) W \\
& +\left(-\alpha_{1}+\sin (\theta) \cos ^{2}(\theta) \tilde{a}_{2} \alpha_{1}-10 \cos ^{2}(\theta) \alpha_{1}-\cos (\theta) \sin (\theta) \alpha_{2} \omega\right) r^{2}-3 W^{2} \alpha_{1} \\
& +\left(\cos ^{3}(\theta) \sin (\theta) \tilde{a}_{2}^{2}-8 \cos ^{4}(\theta) \tilde{a}_{2} \tilde{b}-8 \sin (\theta) \cos (\theta) \tilde{b}-80 \cos ^{3}(\theta) \sin (\theta) \tilde{b}\right. \\
& -100 \cos ^{3}(\theta) \sin (\theta)+9 \cos ^{2}(\theta) \tilde{a}_{2}-20 \cos 4(\theta) \tilde{a}_{2}+8 \cos ^{2}(\theta) \tilde{a}_{2} \tilde{b} \\
& -10 \sin (\theta) \cos (\theta)) W r^{2}+\left(-30 \sin (\theta) \cos (\theta)-24 \sin (\theta) \cos (\theta) \tilde{b}-3(\cos (\theta))^{2} \tilde{a}_{2}\right) W^{3} .
\end{aligned}
$$

Hence, system (13) is transformed into the normal form with respect to the parameter $\epsilon$ in order to apply the averaging method. We now consider the averaging differential system of first order for system (13). Using Theorem 2 , we note that all the assumptions of Theorem 2 are satisfied for the differential system (13), where we put $t=\theta, T=2 \pi, x=$ $(r, W) \in(0, \infty) \times \mathbb{R}, x_{0}=\left(r_{0}, W_{0}\right)$ and

$$
f_{1}(t, x)=f_{1}(\theta, r, W)=\left[\begin{array}{l}
f_{1,1}(\theta, r, W) \\
f_{1,2}(\theta, r, W)
\end{array}\right]
$$

Now we denote $g(r, W)=\left(g_{11}(r, W), g_{12}(r, W)\right)$ and compute the integrals (6), i.e. the averaged functions with $y=(r, W)^{T}$,

$$
g(r, W)=\left[\begin{array}{l}
g_{1,1}(r, W) \\
g_{1,2}(r, W)
\end{array}\right]=\left[\begin{array}{c}
\frac{1}{2 \pi} \int_{0}^{2 \pi} f_{1,1} d \theta \\
\frac{1}{2 \pi} \int_{0}^{2 \pi} f_{1,2} d \theta
\end{array}\right]=\left[\begin{array}{c}
-\frac{r W}{\omega}(3+4 \tilde{b}), \\
\frac{3}{\omega}\left(2 r^{2}-\alpha_{4}^{2}+W^{2}\right)
\end{array}\right] .
$$

Therefore, the $g_{11}(r, W),=g_{12}(r, W)=0$ has a unique real solution $\left(r^{*}, W^{*}\right)$ where

In addition, to compute the Jacobian we have

$$
\left(r^{*}, W^{*}\right)=\left(\frac{1}{\sqrt{2}} \sqrt{\alpha_{4}^{2}}, 0\right) \neq 0 .
$$

$$
\frac{\partial g(r, W)}{\partial(\mathrm{r}, \mathrm{W})}=\left[\begin{array}{cc}
\frac{\partial g_{1,1}}{\partial r} & \frac{\partial g_{1,1}}{\partial W} \\
\frac{\partial g_{1,2}}{\partial r} & \frac{\partial g_{1,2}}{\partial W}
\end{array}\right]=\left[\begin{array}{cc}
-\frac{W}{\omega}(3+4 \tilde{b}) & -\frac{r}{\omega}(3+4 \tilde{b}) \\
\frac{12 \mathrm{r}}{\omega} & \frac{6 \mathrm{~W}}{\omega}
\end{array}\right]
$$

That is, the Jacobian matrix of (14) at the point $\left(r^{*}, W^{*}\right)$ takes the value

$$
\left|\frac{\partial g(r, W)}{\partial(\mathrm{r}, \mathrm{W})}\right|_{\left(\frac{1}{\sqrt{2}} \sqrt{\alpha_{4}^{2}}, 0\right)}=\frac{6 \alpha_{4}^{2}(3+4 \tilde{b})}{\omega^{2}}
$$


which is different from zero where $\tilde{b} \neq-\frac{3}{4}$ and $\alpha_{4} \neq 0$, and the eigenvalues at the solution $\left(r^{*}, W^{*}\right)$ are

$$
\pm \frac{1}{\omega} \sqrt{-6 \alpha_{4}^{2}(3+4 \tilde{b})}
$$

These eigenvalues are the same as given in (3). This shows that when

$$
\tilde{b}<-\frac{3}{4}
$$

the periodic solution is unstable, and it has a stable manifold and an unstable manifold both formed by two cylinders.

From the averaging theorm (Theorem 2), the proof is deduced once we show that a periodic solution corresponding to $\left(r^{*}, W^{*}\right)$ produces a periodic solution which bifurcates from the origin of system (8) when $\epsilon=0$.

Theorem 2 guarantees that, for $\epsilon>0$ sufficiently small, there is a periodic solution corresponding to the point $\left(r^{*}, W^{*}\right)$ of the form $(r(\theta, \epsilon), W(\theta, \epsilon))$ such that $(r(0, \epsilon), W(0, \epsilon)) \rightarrow\left(r^{*}, W^{*}\right)$ when $\epsilon$ approches to zero. So, the differential system (11) possess a periodic solution

$$
(U(\theta, \epsilon), V(\theta, \epsilon), W(\theta, \epsilon))=(r \cos (\theta), r \sin (\theta), W),
$$

for $\epsilon>0$ sufficiently small. Consequently, under the change of variables (10) the differntial system (9) posesses the periodic solution $(X(\theta), Y(\theta), Z(\theta))$ obtained from the relation (16). Finally, for $\epsilon>0$ sufficiently small, system (8) possesses the periodic solution

$$
(x(\theta), y(\theta), z(\theta))=(\epsilon X(\theta), \epsilon Y(\theta), \epsilon Z(\theta))
$$

tending to the origin of coordinates when $\epsilon$ approches to zero. Then, it is the periodic solution which starts at the zeroHopf equilibrium point located at the origin when $\epsilon$ is equal to zero. This completes the proof.

\section{Conclusion}

In this article, we have considered the Generalized Stretch-Twist-Fold Flow (GSTF) system flow orginally suggested by Jianghong and Qigui. They added two extra parameters in the STF flow in order to further investigate the STF system. We have then described the values of the parameters for which a zero-Hopf equilibrium occurs at the origin for this system. We showed that there is only one condition for parameters of this type of system (Eq. 1) for which the equilibrium point is a zero-Hopf equilibrium. Moreover, we have used the averaging method of first order (see Theorem 2) to determine a periodic solution, which bifurcates from the zero-Hopf equilibrium point, see Theorem 1. This theorem gives a periodic solution of the GSTF. It follows from theorm 2 that any sufficiently small system has a periodic solution of period near $2 \pi$. Clearly this periodic orbit tends to the origin of coordinates when tends to zero. Therefore, this is the small amplitude periodic solution, which starts at the zero-Hopf equilibrium.

\section{Conflict of interest}

The authors delcare no conflict of interest.

\section{Acknowledgment}

We would like to express our gratitude to the referees for their comments and valuable suggestions to improve the presentation of this work.

\section{References}

1. Vainshtein, S.I. and Zel'dovich, Y.A. Origin of magnetic fields in astrophysics (turbulent "dynamo" mechanisms). Soviet Physics Uspekhi, 1972, 15(2), 159-172.

2. Moffatt, H.K. Stretch, twist and fold. Nature, 1989, 341(6240), 285-286.

3. Childress, S. and Gilbert, A.D. Stretch, Twist, Fold: The Fast Dynamo. Springer, Berlin/Heidelberg, 1995.

4. Bajer, K. and Moffatt, H. On a class of steady confined stokes flows with chaotic streamlines. Journal of Fluid Mechanics, 1990, 212, 337-363.

5. Samuel, I.V., Roald, Z.S., Rosner, R. and Kim, E. Fractal properties of the stretch-twist-fold magnetic dynamo. Physical Review E, 1996, 53(5), 4729-4744.

6. Jianghong, B. and Qigui, Y. Complex dynamics in the stretch-twist-fold flow. Nonlinear Dynamics, 2010, 61(4), 773-781.

7. Jianghong, B. and Qigui, Y. A new method to find homoclinic and heteroclinic orbits. Applied Mathematics and Computation, 2011, 217(14), 6526-6540. 
8. Jianghong, B. and Qigui, Y. Bifurcation analysis of the generalized stretch-twist-fold flow. Applied Mathematics and Computation, 2014, 229, 16-26.

9. Jianghong, B. and Qigui, Y. Darboux integrability of the stretch-twist-fold flow. Nonlinear Dynamics, 2014, 76(1), 797-807.

10. Buzzi, C., Llibre, J. and Medrado, J. Hopf and zero-Hopf bifurcations in the Hindmarsh-Rose system. Nonlinear Dynamics. 2016, 83(3), 1549-1556.

11. Junze, L., Yebei L. and Zhouchao W. Zero-Hopf bifurcation and Hopf bifurcation for smooth Chua's system. Advances in Difference Equations, 2018, 1, 141-158.

12. Llibre, J. Periodic orbits in the zero-hopf bifurcation of the Rossler system. Roman. Astron. J., 2014, 24(1), 4960 .

13. Llibre, J., Oliveira, R.D.S. and Valls, C. On the integrability and the zero-Hopf bifurcation of a Chen-Wang differential system. Nonlinear Dynamics, 2015, 80(1-2), 353-361.

14. Barreira, L., Llibre, J. and Valls, C. Limit cycles bifurcating from a zero-Hopf singularity in arbitrary dimension, Nonlinear Dynamics. 2018, 92, 1159-1166.

15. Llibre, J. and Amar M. Zero-Hopf bifurcation in the generalized Michelson system. Chaos, Solutions and Fractals, 2016, 89, 228-231

16. Llibre, J. and Perez-Chavela, E. Zero-Hopf bifurcation for a class of Lorenz-type systems. Discrete Continuous Dynamical Systems-Series B, 2014, 19(6), 1731-1736. Llibre, J. and Rodrigo D. Zero-Hopf bifurcation in a Chua system. Nonlinear Analysis: Real World Applications, 2017, 37, 31-40.

17. Guckenheimer, J. On a Codimension two bifurcation, in: Dynamical Systems and Turbulence. Warwick, 1980, Springer, 1981, 99-142.

18. Guckenheimer, J. and Holmes, P. Nonlinear oscillations, dynamical systems, and bifurcations of vector fields, 2013, 42. Springer Science and Business Media, 2013.

19. Scheurle, J. and Marsden, J. Bifurcation to quasi-periodic tori in the interaction of steady state and hopf bifurcations, SIAM Journal on Mathematical Analysis, 1984, 15(6), 1055-1074

20. Kuznetsov, Y.A. Elements of applied bifurcation theory, Vol. 112, Springer Science and Business Media, 2013.

21. Baldom'a, I. and Seara, T. Brakdown of heteroclinic orbits for some analytic unfoldings of the Zero-Hopf singularity, Journal of Nonlinear Science., 2006, 16, 543-582.

22. Broer, H. and Vegter, G., Subordinate Silnikov bifurcations near some singularities of vector fields having low codimension, Ergodic Theory Dynamic Systems, 1984, 4, 509-525.

23. Fatou, P. On the movement of a system subject to short-period forces. Bulletin of the Mathematical Society of France. 1928, 56, 98-139.

24. Bogoliubov, N. and Krylov, N. The application of methods of nonlinear mechanics in the theory of stationary oscillations, Publication 8 of Ukrainian Academy of Sciences, Kiev, 1934.

25. Bogolyubov, N. On some statistical methods in mathematical physics, Izdat. Akad. Nauk Ukr. SSR, Kiev. 1945.

26. Sanders, J.A. and Verhulst, F. Averaging Methods in Nonlinear Dynamical Systems, in: Applied Mathematics Science, Vol. 59, Springer, 1985.

Received 23 October 2018

Accepted 31 January 2019 